\title{
Imaging Psoas Sign in Lumbar Spinal Infections: Evaluation of Diagnostic Accuracy and Comparison with Established Imaging Characteristics
}

\author{
(D).N. Ledbetter, DK.L. Salzman, and (D)L.M. Shah
}

\begin{abstract}
BACKGROUND AND PURPOSE: Lumbar discitis-osteomyelitis has imaging characteristics than can overlap with noninfectious causes of back pain. Our aim was to determine the added accuracy of psoas musculature T2 hyperintensity (imaging psoas sign) in the MR imaging diagnosis of lumbar discitis-osteomyelitis.
\end{abstract}

MATERIALS AND METHODS: This retrospective case-control study evaluated lumbar spine MR imaging examinations, during a 30-month period, that were requested for the evaluation of discitis-osteomyelitis. Of this pool, 50 age-matched control patients were compared with 51 biopsy-proved or clinically diagnosed patients with discitis-osteomyelitis. Two reviewers, blinded to the clinical information, assessed the randomly organized MR imaging examinations for abnormalities of the psoas musculature, vertebral bodies, discs, and epidural space.

RESULTS: Psoas T2 hyperintensity demonstrated a high sensitivity (92.1\%; 95\% Cl, 80\%-97.4\%) and specificity (92\%; 95\% Cl, 80\%-97.4\%), high positive likelihood ratio $(11.5 ; 95 \% \mathrm{Cl}, 4.5-29.6)$, low negative likelihood ratio $(0.09 ; 95 \% \mathrm{Cl}, 0.03-0.20)$, and individual area under the receiver operating characteristic curve of $0.92 ; 95 \% \mathrm{Cl}, 0.87-0.97$. Identification of psoas $\mathrm{T} 2$ abnormality significantly improved $(P=.02)$ the diagnostic accuracy of discitis-osteomyelitis in noncontrast examinations from an area under the receiver operator characteristic curve of the established variables (vertebral body T2 and Tl signal, endplate integrity, disc T2 signal, and disc height) from 0.93 (95\% Cl, 0.88-0.98) to 0.98 (95\% Cl, 0.96-1.0). Psoas T2 abnormalities also had the highest interobserver reliability with a $\kappa$ coefficient of 0.78 (substantial agreement).

CONCLUSIONS: Psoas T2 hyperintensity, the imaging psoas sign, is highly correlated with discitis-osteomyelitis. T2 hyperintensity in the psoas musculature, particularly when there is clinical suspicion of spinal infection, improves the diagnostic accuracy of discitis-osteomyelitis compared with routine noncontrast variables alone.

ABBREVIATIONS: $A U C=$ area under the receiver operating characteristic curve; $D O M=$ discitis-osteomyelitis; LBP = low back pain

$D^{i}$ iscitis-osteomyelitis (DOM) is a significant cause of morbidity and mortality in patients with low back pain (LBP). ${ }^{1-3}$ It can be a diagnostic challenge due to overlapping symptoms and imaging findings with other noninfectious causes of LBP. MR imaging is the standard technique for LBP evaluation, particularly when there is a clinical concern for infection, because of the superb soft-tissue resolution. ${ }^{4-9}$

Several MR imaging characteristics are associated with spinal

Received July 17, 2015; accepted after revision August 21.

From the Department of Radiology (L.N.L.), University of Kansas Medical Center, Kansas City, Kansas; and Department of Radiology (K.L.S., L.M.S.), University of Utah, Salt Lake City, Utah.

Paper previously presented at: Annual Meeting of Radiological Society of North America, November 30 -December 5, 2014; Chicago, Illinois.

Please address correspondence to Luke Ledbetter, MD, University of Kansas, Department of Radiology, 3901 Rainbow Blvd MS 4032, Kansas City, KS 66160; e-mail: lledbetter@kumc.edu; @LNLedbetter

三 Indicates article with supplemental on-line tables.

http://dx.doi.org/10.3174/ajnr.A4571 infections, including vertebral body low T1 and high T2 signal, intervertebral disc high $\mathrm{T} 2$ signal, endplate destruction, and vertebral body and intervertebral disc enhancement. ${ }^{9-14}$ Unfortunately, these MR imaging features can be seen with noninfectious causes of LBP such as disc degeneration, reactive endplate changes, inflammatory spondyloarthopathy, spinal neuroarthropathy, hemodialysis-related spondyloarthopathy, and neoplastic conditions such as chordoma, lymphoma, or metastasis. ${ }^{15-18}$ Although intravenous contrast is often used to help differentiate DOM from the aforementioned conditions, the spinal inflammatory process can be confounding. Furthermore, patients with contraindications to contrast or patients with vague symptoms of LBP often receive noncontrast examinations, and the diagnosis can be difficult.

Paraspinal soft-tissue abnormalities on MR imaging may be overlooked due to the focus on the discovertebral complex or may be referenced as an adjunct finding in spinal infections. However, these structures may provide critical information that is the diagnostic key. 
Previous studies have considered all abnormalities of the paravertebral region as a single variable, inclusive of the anterior, lateral, and posterior (epidural) spaces. DOM begins along the subchondral endplates in adults; and given the close proximity of the psoas musculature to the anterolateral endplates and discs, the signal intensity changes in the psoas can be useful in differentiating infectious and noninfectious etiologies of low back pain.

The purpose of this retrospective case-control study was to review the accuracy of MR imaging characteristics in lumbar spinal infections, particularly evaluating psoas musculature T2 hyperintensity (imaging psoas sign) in the diagnosis of discitis-osteomyelitis.

\section{MATERIALS AND METHODS \\ Patient Selection}

After approval by the institutional internal review board, a retrospective review of the radiology information system data base queried for lumbar spine MR imaging reports with the key terms "infection," "discitis," "osteomyelitis," and "spondylodiscitis" from January 2011 to July 2014. This search captured all studies either performed with the clinical question of infection or with imaging findings that warranted the radiologist mentioning infection in the report. Each patient's electronic medical record was reviewed for presentation, comorbidities, clinical course, biopsy results, and treatment to create infection and control groups. The infection group included patients with biopsy-proved infection and/or the clinical diagnosis and treatment of DOM. The clinical diagnosis was defined by evaluation, diagnosis, treatment, and follow-up of DOM by an infectious disease physician. The control group was randomly selected from the initial radiology information system search for subjects of similar ages and without the imaging or clinical diagnosis of DOM, as defined above. Patients were excluded if there was lack of clinical information or infectious disease diagnosis, if they had isolated sacral infection, if they had an anterior or lateral approach for spinal fusion involving the psoas musculature, and if they had incomplete or nondiagnostic imaging studies. Postoperative patients treated via a posterior approach were included in the study because newer MR imaging techniques such as Dixon and Warp minimize susceptibility artifacts and enable adequate assessment of the osseous spine and adjacent soft tissues.

\section{Image Evaluation}

MR imaging examinations were performed on a 1.5T scanner (Magnetom Avanto or Aera; Siemens, Erlangen, Germany). Sagittal T1weighted inversion recovery (T1-FLAIR) and T2-weighted images were reviewed (T1 inversion recovery: 3-mm section thickness; TR, $2000 \mathrm{~ms}$; TE, $10 \mathrm{~ms}$; TI, $830 \mathrm{~ms}$; FOV, $22 \mathrm{~cm}$; T2: 3-mm section thickness; TR, 3000-6000 ms; TE, 112 ms; FOV, 22 cm). Axial T1 and $\mathrm{T} 2$ images were also reviewed (T1: 4-mm section thickness; TR, $506 \mathrm{~ms}$; TE, $16 \mathrm{~ms}$; FOV, $18 \mathrm{~cm}$; T2: 4-mm section thickness; TR, 4000-5000 ms; TE, $106 \mathrm{~ms}$; FOV, $18 \mathrm{~cm}$ ). Sagittal and axial T1 postcontrast images (sagittal: 3-mm section thickness; TR, 464 ms; TE, 14 ms; FOV, 22 cm; axial: 4-mm section thickness; TR, 508 ms; TE, 16 $\mathrm{ms}$; FOV, $18 \mathrm{~cm}$ ) were reviewed when available. Gadobenate dimeglumine (MultiHance; Bracco Diagnostics, Princeton, New Jersey) was administered at a dose of $0.1 \mathrm{~mL} / \mathrm{kg}$ (standard dose of $20 \mathrm{~mL}$ ) for contrast-enhanced sequences.

The study and control patients were randomized alphabeti- cally on the PACS. Two neuroradiologists (L.M.S., K.L.S.) with Certificates of Added Qualification in neuroradiology and $>10$ years' experience were blinded to the final diagnosis and the clinical course and separately reviewed MR imaging examinations. MR imaging characteristics were evaluated categorically at the level of greatest signal abnormality relative to adjacent levels. Those cases discordant between the reviewers were subject to blinded group re-review to achieve consensus.

Intervertebral discs were graded on disc height, T2 signal, and enhancement. ${ }^{13}$ Disc height was graded as normal, loss of $<50 \%$, loss of $>50 \%$, or increased. T2 signal was graded as isointense, hyperintense, or hypointense. Enhancement was graded as absent, focal, rim, or diffuse.

Vertebral bodies were graded on T1 signal, T2 signal, endplate integrity, and enhancement. ${ }^{13} \mathrm{~T} 1$ and T2 signals were graded as isointense, hyperintense, or hypointense. Endplate integrity was evaluated on T1-weighted images and graded as intact, eroded with normal marrow signal, or destroyed with abnormal marrow signal. Enhancement was graded as absent, endplate, or diffuse.

Epidural space was evaluated for the presence or absence of phlegmon and abscess. The psoas musculature was evaluated for the presence or absence of abnormal T2 signal, enhancement, and abscess.

\section{Statistical Analysis}

Descriptive test characteristics (sensitivity, specificity, positive and negative likelihood ratios) are reported with 2-sided 95\% confidence intervals ${ }^{19}$ for each of the different MR imaging variables described previously. Statistical significance between the spinal infection and control groups was determined by using a 2-tailed Fisher exact test. Interobserver agreement was calculated by using a $\kappa$ coefficient. $^{20,21}$ Multivariate logistic regression for the noncontrast variables alone and with the psoas T2 hyperintensity was performed to obtain $P$ values, area under the receiver operating characteristic curve (AUC), and confidence intervals. The AUCs for the 2 regression models were compared for significance. Statistical significance was defined as a $P$ value $<.05$. STATA statistical software: Release 14 (StataCorp, College Station, Texas) was used for all statistical analysis.

\section{RESULTS}

\section{Demographics}

Of the 205 patients having MR imaging of the lumbar spine with reports containing the key words "infection," "discitis," "osteomyelitis," or "spondylodiscitis, " 51 patients were categorized in the DOM group after the initial imaging and chart review. There were 154 patients from this data search eligible for the control group who did not have the final diagnosis of DOM, and 50 patients from this group were randomly selected for the final control group (Table 1). We excluded 9 of 205 patients: 5 without available clinical or imaging follow-up, 2 with isolated sacral infections, 1 with nondiagnostic imaging, and 1 with muscular metastatic disease. No patients had previous anterior- or lateralapproach spinal surgical procedures.

The DOM group included 40 patients who had image-guided biopsies of the suspected disc and endplate during the course of their evaluation. Percutaneous biopsy of the disc and endplate was performed in 38 of the 40 biopsies with the remaining 2 un- 
Table 1: Demographics

\begin{tabular}{lcc}
\hline & $\begin{array}{c}\text { Spinal Infection } \\
(\boldsymbol{n}=\mathbf{5 1})\end{array}$ & $\begin{array}{c}\text { Controls } \\
(\boldsymbol{n}=\mathbf{5 0})\end{array}$ \\
\hline Age (yr) (range) & $58.8(19-87)$ & $53.6(18-93)$ \\
Sex & 41 Male, 10 female & 25 Male, 25 female \\
Previous spinal surgery & 12 & 6 \\
Contrast-enhanced exams & 45 & 39 \\
Non-contrast-enhanced & 6 & 11 \\
exams & & \\
\hline
\end{tabular}

dergoing surgical biopsy. Two of the 38 patients with percutaneous disc biopsies also included samples of the paraspinal soft tissues. Microbiologic or histopathologic findings consistent with infection were identified in 34 of 40 patients $(85 \%)$. The most common isolated pathogen was Staphylococcus aureus (13 patients), followed by Enterococcus faecalis (5 patients), Propionibacterium acnes (3 patients), multiple pathogens (3 patients), and 1 patient each with Staphylococcus epidermidis, viridans streptococci, Finegoldia magna, Enterococcus coli, group B streptococcus, $M y$ cobacterium tuberculosis, and Streptococcus mitis. Three patients had histologic findings of infection without positive microbiology cultures. Four of the 6 patients with a biopsy negative for infection received intravenous antibiotic treatment before biopsy. All 11 patients who were not biopsied had bacteremia, 9 with $S$ aureus, 1 with E faecalis, and 1 with Klebsiella pneumoniae. The 6 patients with biopsy results negative for infection, together with the 11 patients not biopsied, were diagnosed and treated for spinal infection based on an infectious disease service evaluation and recommendation. Pertinent medical history in these 17 patients included bacteremia, endocarditis, additional septic joints, immunosuppression, and IV drug use. Of the 19 postoperative patients included in our study, 12 were positive for DOM.

\section{Imaging Evaluation}

Results from the blinded review of the cases are listed in On-line Table 1. The level of greatest involvement was T12-L1 in 3 patients, L1-L2 in 5, L2-L3 in 10, L3-L4 in 11, L4-L5 in 11, and $\mathrm{L}-\mathrm{S} 1$ in 11 . The psoas MR imaging features showed statistically significant association with DOM $(P<.001)$. The vertebral body findings with statistical significance included T1 hypointensity, T2 hyperintensity, diffuse enhancement, and endplate destruction and enhancement. Statistically significant disc abnormalities included increased height, T2 hyperintensity, and focal and rim enhancement. Both epidural phlegmon and abscess demonstrated statistically significant association with DOM. Psoas T2 hyperintensity $(P<.001)$ and enhancement $(P<.001)$ showed a statistically significant correlation to DOM in postoperative patients as well.

The findings with the highest sensitivity for DOM included psoas T2 hyperintensity (92.1\%) and vertebral body T1 hypointensity (90.2\%) (Figs 1 and 2). Other findings with high sensitivity included psoas enhancement (88.9\%), vertebral body T2 hyperintensity (88.2\%), and epidural phlegmon (80.9\%). Highly specific findings for DOM included increased disc height and epidural abscess (100\%). Other findings with high specificity included psoas abscess (97.4\%), epidural phlegmon (94.9\%), diffuse vertebral body enhancement (94.5\%), rim enhancement of the disc (92.3\%), psoas T2 hyperintensity (92\%), psoas enhancement
(87.1\%), disc T2 hyperintensity (86.3\%), endplate destruction (86\%), and vertebral body T1 hypointensity (80\%).

The overall diagnostic accuracy of the individual MR imaging variables to distinguish the infection and control groups was evaluated by receiver operating characteristic analysis (On-line Table 1). High T2 signal within the psoas musculature had the highest individual AUC of 0.92. Other variables with the high AUC included psoas enhancement (0.88), epidural phlegmon (0.88), and vertebral body $\mathrm{T} 1$ hypointensity $(0.85)$.

Positive and negative likelihood ratios were calculated to demonstrate the potential utility of the measured imaging characteristics in DOM (On-line Tables 2 and 3). High positive likelihood ratios, which indicate an increase in the probability of disease with a positive result, were identified with epidural phlegmon (15.8), psoas abscess (14.9), diffuse vertebral body enhancement (12.1), and psoas T2 hyperintensity (11.5). Low negative likelihood ratios, indicating a lower probability of infection with a test with negative findings, were identified with psoas T2 hyperintensity (0.09), T1 vertebral body hypointensity $(0.12)$, psoas enhancement (0.13), and vertebral body T2 hyperintensity (0.16).

Multivariate logistic regression analysis of the noncontrast variables, with and without psoas T2 hyperintensity, was performed to determine the diagnostic accuracy and significance of the psoas T2 variable. The total AUC of the combination of routine noncontrast variables (vertebral body $\mathrm{T} 2$ and $\mathrm{T} 1$ signal, endplate integrity, disc T2 signal, and disc height) was 0.93 (95\% CI, $0.88-0.98)$. The addition of psoas $\mathrm{T} 2$ hyperintensity to these variables resulted in an AUC of 0.98 (95\% CI, 0.96-1.00), which was statistically significant $(P=.002)$.

Psoas abnormalities were infrequently seen in patients with LBP who were not subsequently diagnosed with spinal infection. Only 4 of 50 patients $(8 \%)$ had psoas abnormalities in the control group. These findings were related to myositis $(n=2)$, noninfectious spondylodiscitis (Anderssen lesion) in ankylosing spondylitis $(n=1)$, and extension of inflammation from an iliacus abscess $(n=1)$.

\section{Interobserver Agreement}

$\kappa$ coefficient values were calculated on the initial blinded review (Table 2). Agreement was almost perfect in ratings of psoas abscess (0.87) and enhancement (0.81). There was substantial agreement with psoas T2 hyperintensity, vertebral body T2 hyperintensity, T1 hypointensity and enhancement, disc T2 hyperintensity, and endplate integrity. Moderate agreement in ratings included epidural phlegmon and abscess as well as disc enhancement and increased height.

\section{DISCUSSION}

DOM often presents with nonspecific indolent clinical symptoms but can have potentially devastating complications, which make imaging essential for early diagnosis. Additionally, the incidence of spinal infections has increased during the past 2 decades and has transitioned from an acute high mortality process to a more indolent chronic debilitating disease. ${ }^{1}$ Early diagnosis is key in preventing adverse outcomes that are common in advanced disease. ${ }^{2}$ However, imaging findings in spinal infections can be nonspecific and overlap other causes of noninfectious LBP, especially on noncontrast examinations. ${ }^{16,22}$ 

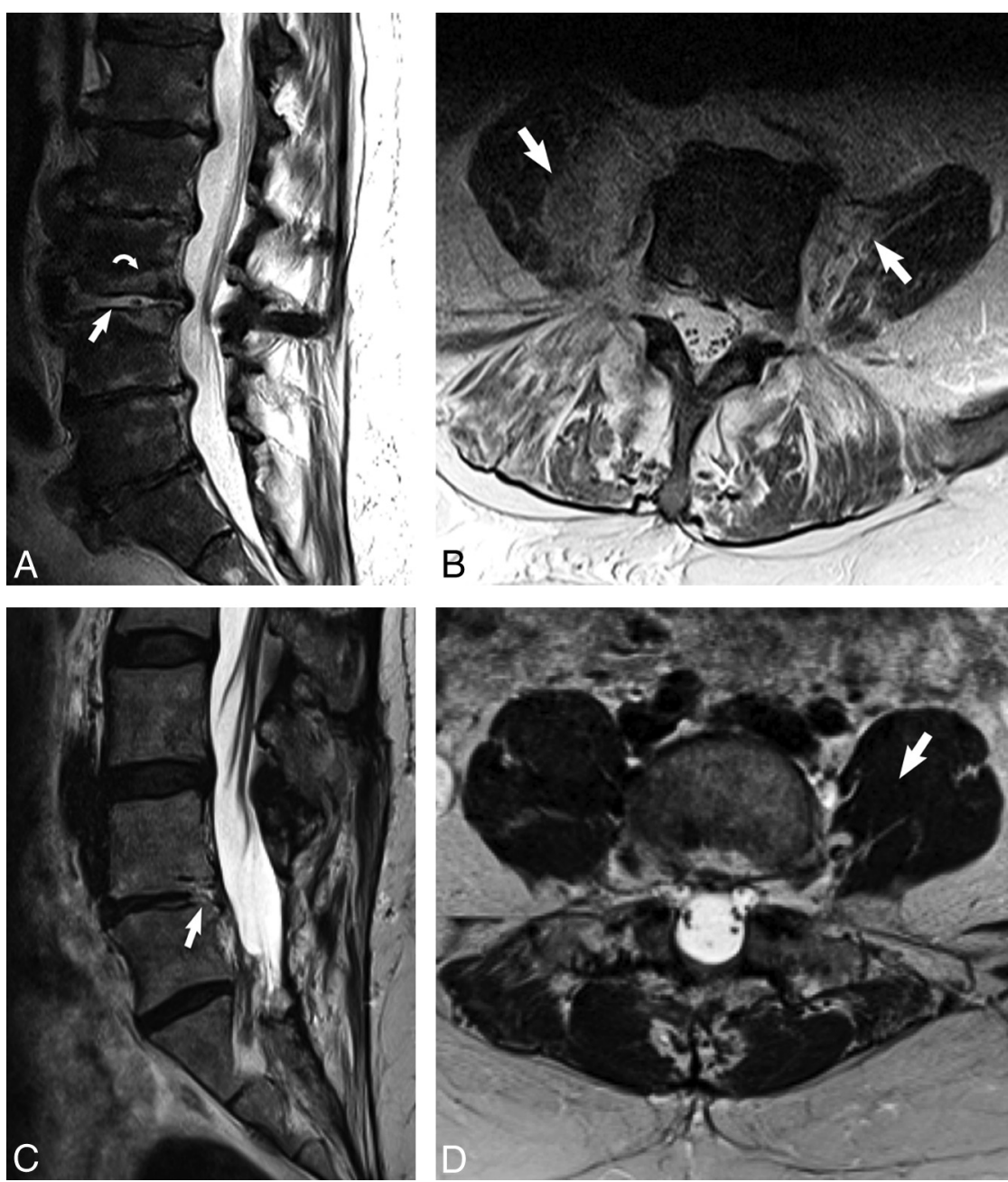

FIG 1. The imaging psoas sign on noncontrast MR imaging of the lumbar spine in an 81-year-old man with renal failure, $E$ faecalis bacteremia, and clinically diagnosed discitis-osteomyelitis ( $A$ and $B$ ) with comparison with degenerative disc disease $(C$ and $D)$. $A$, Sagittal $T 2$ image demonstrates high $T 2$ signal within the disc (white arrow) and the adjacent endplates (white curved arrow) in the patient with discitis-osteomyelitis. B, Axial T2 image at the level of $L 3-L 4$ of the same patient demonstrates the imaging psoas sign of T2 hyperintensity within the bilateral psoas musculature (white arrows). C, Sagittal T2 image in a patient with degenerative disc disease shows focal high T2 signal involving the posterior endplate and disc of L4-L5 (white arrow). D, Axial T2 image in the same patient at the level of L4 L L5 shows normal and uniform T2 signal within the psoas muscles (white arrow).

In addition, biopsy with microbiologic and histopathologic evaluation has limitations, often with poor diagnostic yield and a wide range of accuracies in organism identification or structural changes consistent with infection. ${ }^{23}$ Frequently, the combination of clinical, imaging, and laboratory evaluation leads to a treatment regimen without a definitive finding on any level of investigation.

The purpose of this study was to determine whether there are specific imaging features that improve the MR imaging diagnosis of lumbar discitis-osteomyelitis.

The results of this study evaluating the diagnostic accuracy of MR imaging characteristics for lumbar DOM demonstrate that abnormal T2 signal intensity in the psoas musculature, the imaging psoas sign, is essential and improves diagnostic accuracy on noncontrast examinations over the standard noncontrast variables alone (Figs 1 and 2). Psoas T2 hyperintensity had the highest individual sensitivity (92.1\%), specificity (92\%), and AUC (0.92) in the evaluation of DOM. Its high positive and low negative likelihood ratios (11.5 and 0.09 respectively) suggest increased suspicion of DOM with a positive result and decreased suspicion with a negative result. The imaging psoas sign showed excellent interrater reliability. Even in postoperative studies with the challenge of discerning DOM from expected surgical changes, psoas $\mathrm{T} 2$ hyperintensity demonstrated a statistically significant association with DOM compared with the noninfected patients postoperatively. False-positive findings of T2 abnormalities of the psoas muscle occurred infrequently in the control population $(8 \%)$. Conditions that resulted in false-positive findings included 2 cases of myositis in immunocompromised patients, an Andersson lesion of ankylosing spondylitis, iatrogenic softtissue edema after LP, and extension of a large iliacus abscess into the psoas muscle. The small but potential risk of a type I error may be reduced by incorporating all imaging and clinical information when making the diagnosis of DOM.

Abnormalities of the paravertebral soft tissues, largely considered in the literature as a combination of prevertebral and epidural spaces, have been described as highly sensitive for spinal infection. ${ }^{13}$ Specifically, epidural abscess and phlegmon have known associations with spinal infections. ${ }^{22,24}$ To our knowledge, this is first study to evaluate the MR imaging appearance of the psoas musculature, specifically as an indicator of lumbar DOM. Psoas abscess has been associated with a spinal source of infection $^{25,26}$ but often occurs as a late presentation of disease. However, this study addresses the significance of psoas signal abnormalities on nonenhanced sequences. This is important because many patients with DOM do not receive IV contrast on the initial MR imaging evaluation either due to contraindication to contrast or the nonspecific presenting symptoms.

The psoas muscles originate from the transverse processes of all the lumbar vertebral bodies and the anteromedial lumbar discs and adjacent endplates. ${ }^{24}$ Given that DOM is believed to commonly originate from the anterior endplates, ${ }^{15,27}$ the psoas muscle is in a location that is affected early in the infectious process by direct spread and potentially earlier than other paraspinous soft tissues, including the epidural space. Psoas abscesses are a delayed manifestation of infection ${ }^{25,26}$; therefore, early identification T2 signal changes in the psoas musculature can potentially help detect DOM early in the course and potentially prevent long-term complications. 

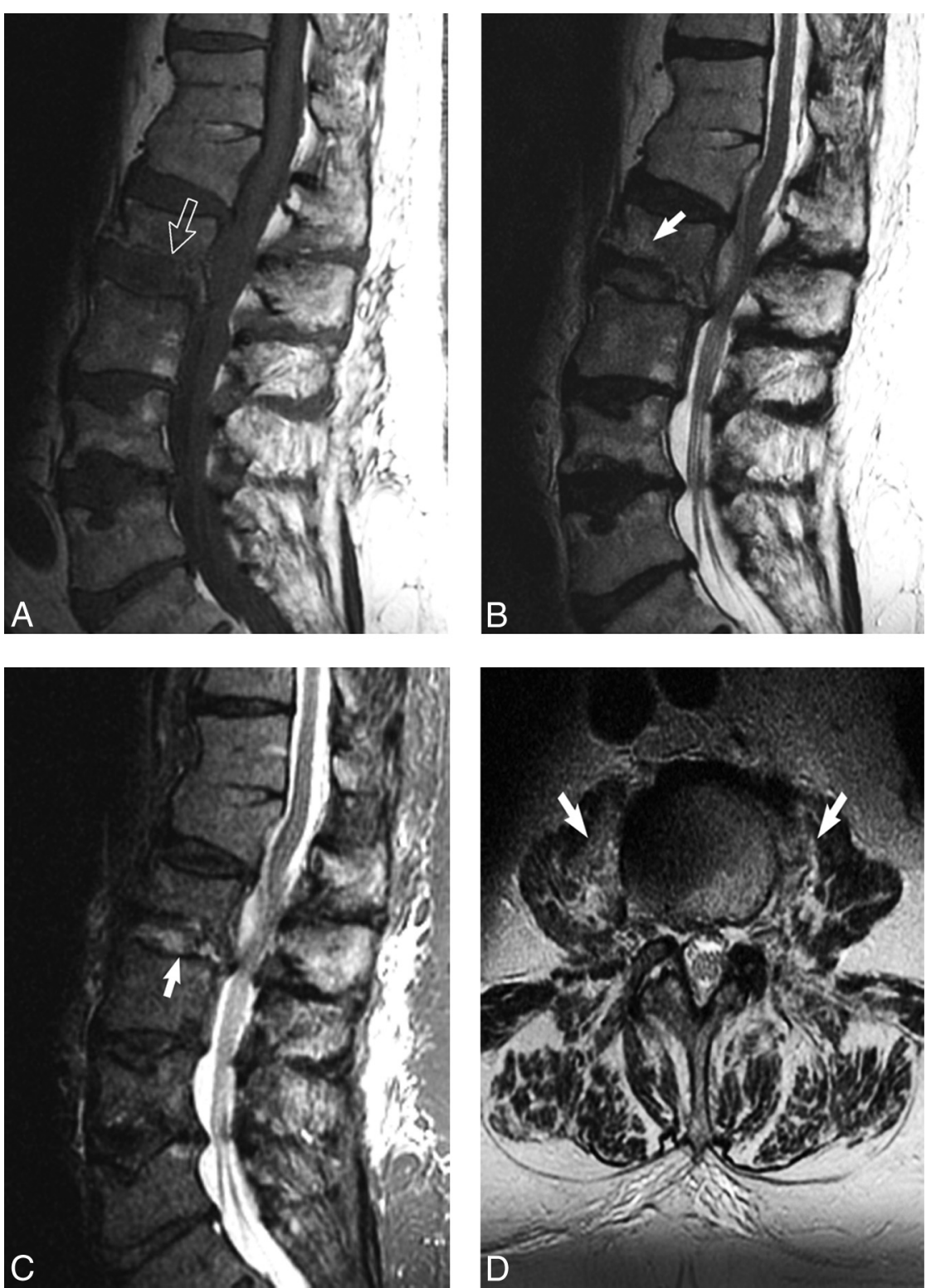

FIG 2. The imaging psoas sign in biopsy-proved $S$ aureus discitis-osteomyelitis in a 79-year-old man with history of long-term steroid treatment of hypersensitivity pneumonitis. A, Sagittal T1 image shows height loss of L2 with associated increased L2-L3 disc space height. Normal T1 hypointensity is absent along the inferior $\mathrm{L} 2$ endplate due to destruction (white open arrow). $B$, Sagittal T2 image demonstrates subtle L2 marrow T2 hyperintensity (white arrow). C, Sagittal STIR image shows increased conspicuity of T2 hyperintensity within the L2-L3 disc space (white arrow). D, Axial T2 image at the level of the superior L3 vertebral body demonstrates bilateral psoas muscle hyperintense signal, the imaging psoas sign (white arrows).

Abnormalities of the disc space and vertebral bodies, including high T2 intradiscal signal, low T1 marrow signal, disc enhancement, and vertebral body enhancement have been shown to be sensitive for DOM. ${ }^{9,12-14}$ Our results agree with these findings with the added evaluation of specificity, receiver operating characteristic analysis, and likelihood ratios. Receiver operating characteristic analysis demonstrated that the vertebral body low T1 signal was the most helpful of the individual nonparaspinous findings in detecting DOM (0.85), followed by vertebral body and disc T2 hyperintensity (both 0.81 ). Increased disc height showed a statistically significant association with DOM, but it had the lowest sensitivity, area under the receiver operating characteristic curve, $\kappa$, and negative likelihood ratio. Disc height was graded as a comparison with adjacent uninvolved discs and may have appeared taller than unaffected discs due to erosion of the endplates, increased fluid within the disc space, or preservation of disc height compared with other degenerated levels.

This study strengthens the evidence that the appearance of the paravertebral soft tissues are critical in the evaluation of DOM and emphasizes the imaging psoas sign as a statistically important variable on MR imaging, specifically on noncontrast examinations. Psoas T2 hyperintensity was best identified on axial T2-weighted images. Although our spine infection MR imaging protocol does not use fat-saturation techniques on the axial T2-weighted sequences, the addition of the fat saturation increases the conspicuity of the psoas signal abnormality. This may be useful not only in those patients with LBP as a nonspecific presentation of DOM but especially in those patients with a contraindication to IV contrast. Fat saturation can also increase the conspicuity of equivocal vertebral body and disc space findings. ${ }^{28}$ Future studies could consider the evaluation of MR imaging protocols of different fat-saturation techniques in patients undergoing evaluation for DOM.

Limitations of this study include variations in imaging protocols with a mix of both contrast-enhanced and noncontrast examinations. This study was designed to closely represent the cohort of patients that radiologists encounter in daily practice, in which an imaging interpretation of infection must be made with a pretest clinical suspicion or suspicious imaging findings in patients with a nonspecific presentation. Because the reference standard of microbiologic/histologic confirmation of infection may not have been met in some patients, the clinical diagnosis and treatment for spinal infection was considered as 1 criterion for inclusion into the infection group. The constellation of clinical, imaging, and laboratory evaluations without a definitive finding on any level of investigation often dictates clinical management because biopsy itself is an imperfect diagnostic test with a range of diagnostic yield between $38.1 \%$ and $76.5 \% .{ }^{29-32}$ As such, our results of the high sensitivity and specificity of psoas $\mathrm{T} 2$ hyperintensity will add great diagnostic value. In addition, our case-control cohort is not indicative of the incidence of spinal infection in the general population; therefore, the positive and negative predictive values could not be assessed. An additional pitfall in the psoas sign could include L5-S1 infection. The psoas does not consistently originate from this disc space, 
Table 2: Interobserver reliability

\begin{tabular}{lcl}
\hline & $\boldsymbol{\kappa}$ Coefficient & Agreement \\
\hline Psoas abscess & 0.87 & Almost Perfect \\
Psoas enhancement $^{\text {Psoas T2 }}$ a & 0.81 & Almost Perfect \\
Vertebral body T2 hyperintensity $^{\text {Vertebral body T1 hypointensity }}$ & 0.78 & Substantial \\
Vertebral body enhancement & 0.76 & Substantial \\
Disc T2 hyperintensity & 0.75 & Substantial \\
Endplate destruction & 0.75 & Substantial \\
Epidural phlegmon & 0.67 & Substantial \\
Epidural abscess & 0.59 & Substantial \\
Disc enhancement & 0.58 & Moderate \\
Disc increased height & 0.50 & Moderate \\
\hline
\end{tabular}

a Imaging psoas sign..

and occasionally the muscles may course further anterior in the retroperitoneal space with intervening fat between the muscles and the spinal column. Although not affecting our study group, infection in this location would potentially not involve the psoas until a later presentation of disease, if at all.

\section{CONCLUSIONS}

The imaging psoas sign (high T2 signal in the psoas musculature) is highly correlated with lumbar DOM. In addition to having the highest individual sensitivity and specificity of the nonenhanced features evaluated, the imaging psoas sign also had high interrater agreement. Together with previously established imaging characteristics, including vertebral body $\mathrm{T} 1$ hypointensity, vertebral body and disc T2 hyperintensity, and endplate destruction, the imaging psoas sign significantly improves diagnostic accuracy of DOM on noncontrast MR imaging of the lumbar spine.

Disclosures: Karen L. Salzman—UNRELATED: Royalties: Elsevier, Comments: royalties for books written, most recently Diagnostic Imaging: Brain, 3rd Edition.

\section{REFERENCES}

1. McHenry MC, Easley KA, Locker GA. Vertebral osteomyelitis: longterm outcome for 253 patients from 7 Cleveland-area hospitals. Clin Infect Dis 2002;34:1342-50 CrossRef Medline

2. Mylona E, Samarkos M, Kakalou E, et al. Pyogenic vertebral osteomyelitis: a systematic review of clinical characteristics. Semin Arthritis Rheum 2009;39:10-17 CrossRef Medline

3. Gouliouris T, Aliyu SH, Brown NM. Spondylodiscitis: update on diagnosis and management. J Antimicrob Chemother 2010;65(suppl 3):iiil1-24 CrossRef Medline

4. Modic MT, Pavlicek W, Weinstein MA, et al. Magnetic resonance imaging of intervertebral disk disease: clinical and pulse sequence considerations. Radiology 1984;152:103-11 CrossRef Medline

5. Modic MT, Feiglin DH, Piraino DW, et al. Vertebral osteomyelitis: assessment using MR. Radiology 1985;157:157-66 CrossRef Medline

6. Post MJ, Quencer RM, Montalvo BM, et al. Spinal infection: evaluation with MR imaging and intraoperative US. Radiology 1988;169: 765-71 CrossRef Medline

7. Unger E, Moldofsky P, Gatenby R, et al. Diagnosis of osteomyelitis by MR imaging. AJR Am J Roentgenol 1988;150:605-10 CrossRef Medline

8. Chandnani VP, Beltran J, Morris CS, et al. Acute experimental osteomyelitis and abscesses: detection with MR imaging versus CT. $R a-$ diology 1990;174:233-36 CrossRef Medline
9. Thrush A, Enzmann D. MR imaging of infectious spondylitis. AJNR Am J Neuroradiol 1990;11:1171-80 Medline

10. Meyers SP, Wiener SN. Diagnosis of hematogenous pyogenic vertebral osteomyelitis by magnetic resonance imaging. Arch Intern Med 1991;151:683-87 CrossRef Medline

11. Post MJ, Sze G, Quencer RM, et al. Gadolinium-enhanced MR in spinal infection. J Comput Assist Tomogr 1990;14:721-29 CrossRef Medline

12. Dagirmanjian A, Schils J, McHenry M, et al. MR imaging of vertebral osteomyelitis revisited. AJR Am J Roentgenol 1996;167:1539-43 CrossRef Medline

13. Ledermann HP, Schweitzer ME, Morrison WB, et al. MR imaging findings in spinal infections: rules or myths? Radiology 2003;228: 506-14 CrossRef Medline

14. Patel KB, Poplawski MM, Pawha PS, et al. Diffusion-weighted MRI "claw sign" improves differentiation of infectious from degenerative Modic type 1 signal changes of the spine. AJNR Am JNeuroradiol 2014;35:1647-52 CrossRef Medline

15. Diehn FE. Imaging of spine infection. Radiol Clin North Am 2012;50: 777-98 CrossRef Medline

16. Sharif HS. Role of MR imaging in the management of spinal infections. AJR Am J Roentgenol 1992;158:1333-45 CrossRef Medline

17. Maiuri F, Iaconetta G, Gallicchio B, et al. Spondylodiscitis: clinical and magnetic resonance diagnosis. Spine 1997;22:1741-46 CrossRef Medline

18. Zimmerli W. Clinical practice: vertebral osteomyelitis. N Engl J Med 2010;362:1022-29 CrossRef Medline

19. Brown LD, Cai TT, DasGupta A. Interval estimation for a binomial proportion. Stat Sci 2001;16:101-33 CrossRef

20. Carpenter J, Bithell J. Bootstrap confidence intervals: when, which, what? A practical guide for medical statisticians. Stat Med 2000;19: 1141-64 Medline

21. Reichenheim ME. Confidence intervals for the kappa statistic. Stata J 2004;4:421-28

22. Dunbar JA, Sandoe JA, Rao AS, et al. The MRI appearances of early vertebral osteomyelitis and discitis. Clin Radiol 2010;65:974-81 CrossRef Medline

23. Sehn JK, Gilula LA. Percutaneous needle biopsy in diagnosis and identification of causative organisms in cases of suspected vertebral osteomyelitis. Eur J Radiol 2012;81:940 - 66 CrossRef Medline

24. Lardé D, Mathieu D, Frija J, et al. Vertebral osteomyelitis: disk hypodensity on CT. AJR Am J Roentgenol 1982;139:963-67 CrossRef Medline

25. Jeffrey RB, Callen PW, Federle MP. Computed tomography of psoas abscesses. J Comput Assist Tomogr 1980;4:639-41 CrossRef Medline

26. Mückley T, Schütz T, Kirschner M, et al. Psoas abscess: the spine as a primary source of infection. Spine 2003;28:E106-13 CrossRef Medline

27. Sajko S, Stuber K. Psoas major: a case report and review of its anatomy, biomechanics, and clinical implications. J Can Chiropr Assoc 2009;53:311-18 Medline

28. D'Aprile P, Tarantino A, Lorusso V, et al. Fat saturation technique and gadolinium in MRI of lumbar spinal degenerative disease. $\mathrm{Neu}$ roradiol J 2006;19:654-71 CrossRef Medline

29. Staatz G, Adam GB, Keulers P, et al. Spondylodiskitic abscesses: CT-guided percutaneous catheter drainage. Radiology 1998;208: 363-67 CrossRef Medline

30. Rankine JJ, Barron DA, Robinson P, et al. Therapeutic impact of percutaneous spinal biopsy in spinal infection. Postgrad Med J 2004; 80:607-09 CrossRef Medline

31. D’Agostino C, Scorzolini L, Massetti AP, et al. A seven-year prospective study on spondylodiscitis: epidemiological and microbiological features. Infection 2010;38:102-07 CrossRef Medline

32. Kim BJ, Lee JW, Kim SJ, et al. Diagnostic yield of fluoroscopyguided biopsy for infectious spondylitis. AJNR Am J Neuroradiol 2013;34:233-38 CrossRef Medline 